\title{
ACTIVIDAD FÍSICA EN PACIENTES AMBULATORIOS CON DIABETES MELLITUS 2 DE UN HOSPITAL NACIONAL DEL PERÚ
}

\author{
Ana Josefina Manzaneda ${ }^{1, a}$, María Lazo-Porras ${ }^{1, a}$, Germán Málaga ${ }^{1,2, b}$
}

\begin{abstract}
RESUMEN
Con el objetivo de determinar el nivel de actividad física que realizan los pacientes ambulatorios con diabetes mellitus tipo 2 (DM 2) que acuden a un Hospital Nacional de Lima, Perú se incluyó a 120 pacientes en quienes se aplicó el cuestionario internacional de actividad física IPAQ. El $66 \%$ fueron mujeres, la edad media fue 61,6 años, el $70 \%$ presentó mal control glicémico. El $20 \%$ de pacientes calificaba como inactivo, $68 \%$ con mínima actividad y $12 \%$ con actividad física adecuada. No se halló relación entre actividad física, tiempo de enfermedad, control glicémico, ni el índice de masa corporal. La edad se asoció negativamente con la actividad física. Se concluye que existen bajos niveles de actividad física en pacientes con DM 2 y esta no se enfoca en actividades de ocio que proveen beneficios a la salud.
\end{abstract}

Palabras clave: Diabetes mellitus; Actividad física; Estilo de vida sedentario (fuente: DeCS BIREME).

\section{PHYSICAL ACTIVITY IN OUTPATIENTS WITH TYPE 2 DIABETES IN A NATIONAL HOSPITAL OF PERU}

\begin{abstract}
In order to determine the level of physical activity performed by outpatients with type 2 diabetes seen at a National Hospital in Lima, Peru, we surveyed 120 patients with the International Physical Activity Questionnaire (IPAQ). 66\% were women, the mean age was 61.6 years, and $70 \%$ had poor glycemic control. $20 \%$ of the patients qualified as inactive, $68 \%$ as minimally active, and $12 \%$ had adequate physical activity. No relationship between physical activity, duration of disease, glycemic control, and body mass index was found. Age was negatively associated with physical activity. It is concluded that there are low levels of physical activity in patients with Type 2 diabetes and these are not focused on leisure activities that provide health benefits.
\end{abstract}

Key words: Diabetes mellitus; Motor activity; Sedentary lifestyle (source: MeSH NLM).

\section{INTRODUCCIÓN}

A nivel mundial la incidencia y prevalencia de diabetes mellitus 2 (DM 2) está aumentado debido, entre otros factores, al aumento de la obesidad e inactividad física (1). Se estima que 171 millones de personas en el mundo viven con diabetes y que esta cifra ascenderá a 300 millones en el 2030. El 2013, en el Perú la prevalencia de DM 2 llegó al 5,5\% y la tendencia sigue en aumento (2).

El ejercicio y la actividad física cumplen un rol central en el tratamiento de las enfermedades no transmisibles (ENT), y en particular en la DM $2^{(3,4)}$. La OMS ha determinado que la inactividad física y otros factores de riesgo modificables son responsables del $80 \%$ de las defunciones por ENT ${ }^{(5)}$.
La actividad física es definida como cualquier movimiento corporal producido por los músculos que resultan en gasto energético ${ }^{(6)}$. En el mundo, la inactividad física y el sedentarismo tienen alta prevalencia, supera el $70 \%{ }^{(7-10)}$. Sin embargo, existen discrepancias entre los estudios debido a la diversidad de instrumentos y puntos de cortes inconsistentes ${ }^{(6)}$. Ante esto, la Organización Mundial de la Salud (OMS) ha propuesto al cuestionario internacional de actividad física (IPAQ) como instrumento para vigilancia epidemiológica de la actividad física a nivel poblacional ${ }^{(11)}$.

Debido al incremento de la prevalencia de DM 2, la relevancia de la actividad física en el tratamiento de pacientes con DM 2 cobra importancia. Por ello y por la falta de información a cerca del tema en el Perú, esta

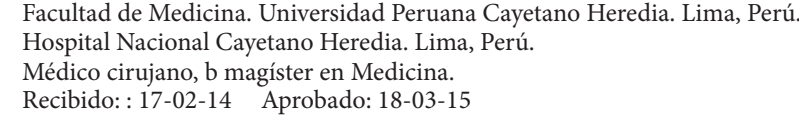


investigación tuvo como objetivo determinar el nivel y el tipo de actividad física que realizaban los pacientes ambulatorios con DM 2, así como las variables clínicas relacionadas con la actividad física, en un Hospital Nacional de Lima, Perú.

\section{EL ESTUDIO}

Se realizó un análisis secundario del estudio de corte transversal: "Calidad del control metabólico en pacientes ambulatorios con diabetes tipo 2 de un hospital general", llevado a cabo entre marzo y agosto de 2012 en el Hospital Nacional Cayetano Heredia, Lima, Perú.

La población del estudio estuvo conformada por pacientes con diagnóstico de DM 2 mayor de un año, que acudieran más de una vez al año a control ambulatorio en consultorio externo del Servicio de Endocrinología del Hospital Nacional Cayetano Heredia, que aceptaron y firmaron el consentimiento para participar en el estudio. Fueron excluidos los pacientes con diagnóstico de diabetes secundaria; coexistencia con enfermedades crónicas no metabólicas; que hubieran estado hospitalizados durante los 6 meses previos a la consulta; por una complicación mayor de DM 2 (cetoacidosis diabética, estado hiperosmolar, accidente cerebro vascular, infarto agudo de miocardio y amputación de pie diabético), y los que no aceptaron participar en el estudio o no firmaron el consentimiento informado.

La muestra estuvo conformada por 120 pacientes, elegidos aleatoriamente. El tamaño de la muestra fue calculado en base al $56 \%$ de control metabólico descrito en pacientes diabéticos ${ }^{(12)}$ con 0,1 de precisión y significancia del 0,05 . Se verificó que la potencia y significancia del estudio no se afectara en el análisis secundario. Se obtuvieron los siguientes datos sociodemográficos, clínicos: tiempo de enfermedad; índice de masa corporal (IMC) y hemoglobina glicosilada (HbA1C), y la actividad física, medida a través del International Physical Activity Questionnaire (IPAQ). EI IPAQ aporta información sobre el gasto energético estimado en 24 horas, y clasifica las actividades de la vida diaria en dominios (laboral, doméstico, transporte y ocio). La actividad física se expresa de manera continua como MET (múltiplos de la tasa metabólica en reposo)minutos/semana, que representan el gasto energético y clasifica de manera categórica el nivel de actividad física en: inactivo, mínimamente activo o activo (Tabla 1$)^{(13)}$.

Se calculó el porcentaje de pacientes inactivos, mínimamente activos e inactivos. Posteriormente, se clasificó a los participantes según su buen control
Tabla 1. Clasificación de los niveles de actividad física según los criterios establecidos por el cuestionario internacional de actividad física (IPAQ)

\begin{tabular}{|c|c|}
\hline $\begin{array}{l}\text { Nivel de } \\
\text { actividad física }\end{array}$ & $\begin{array}{l}\text { Criterios según el cuestionario } \\
\text { internacional de actividad física }\end{array}$ \\
\hline Inactivo & $\begin{array}{l}\text { El nivel de actividad física de } \\
\text { sujeto no está incluido en las } \\
\text { categorías mínimamente activo o } \\
\text { activo. }\end{array}$ \\
\hline \multirow[t]{2}{*}{ Mínimamente activo } & $\begin{array}{l}3 \text { o más días de actividad vigorosa } \\
\text { por al menos } 20 \text { minutos diarios o, } \\
5 \text { o más días de actividad } \\
\text { moderada y/o caminata al menos } \\
30 \text { minutos diarios o, }\end{array}$ \\
\hline & $\begin{array}{l}5 \text { o más de cualquier combinación } \\
\text { de caminata y actividades } \\
\text { moderadas o vigorosas, logrando } \\
\text { al menos } 600 \mathrm{MET} \text {-min/semana }\end{array}$ \\
\hline \multirow[t]{2}{*}{ Activo } & $\begin{array}{l}7 \text { días en la semana de cualquier } \\
\text { combinación de caminata, o } \\
\text { actividades de moderada o alta } \\
\text { intensidad logrando un mínimo de } \\
3000 \text { MET-min/semana o, }\end{array}$ \\
\hline & $\begin{array}{l}\text { Actividad vigorosa al menos } 3 \text { días } \\
\text { a la semana alcanzando al menos } \\
1500 \text { MET-min/semana }\end{array}$ \\
\hline
\end{tabular}

glicémico (HbA1C menor a 7) y mal control glicémico (HbA1C mayor o igual a 7$)^{(12)}$. Además se determinó los MET-minutos/semana (total y según cada dominio) y el estrado nutricional según el índice de masa corporal; normal (IMC menor a 25), sobrepeso (IMC entre 25 y 29,9$)$ y obesidad (IMC mayor a 29,9) ${ }^{(14)}$.

Para el análisis de los datos se utilizó el programa STATA 12.0. Para las variables cuantitativas se calculó la media y desviación stándar en casos de distribución y la mediana para variables con distribución no normal, mientras que para variables cualitativas, se calculó porcentajes. Para comparar medianas se realizó la prueba de Mood y para comparar proporciones utilizamos el test de Chi cuadrado. Se utilizó regresión lineal múltiple para modelar y ajustar por confusores. En todos los casos se consideró que las diferencias eran significativas si el valor de $p \leq 0,05$.

El estudio fue aprobado por el Comité de Ética de la Universidad Peruana Cayetano Heredia, y por el Comité de Ética Hospitalario del Hospital Nacional Cayetano Heredia. Se utilizó un consentimiento informado que fue firmado por los participantes.

\section{HALLAZGOS}

La muestra estuvo constituida por 120 pacientes con DM 2, en su mayoría mujeres $(66 \%)$, la edad promedio fue 61,6 años (SD +/-11,1). El $60 \%$ de los pacientes tuvo 
Tabla 2. Variables sociodemográficas y clínicas de los pacientes con DM 2 atendidos ambulatoriamente en el Hospital Nacional Cayetano Heredia

\begin{tabular}{lc}
\hline Variables sociodemográficas & $\mathbf{n}(\%)$ \\
\hline Edad(años) & $61,6 \pm 11,1$ \\
\hline Mujeres & $79(66)$ \\
Tiempo de enfermedad & \\
$\quad$ Menor de 1 año & $19(14,5)$ \\
Entre 1 y 4 años & $30(24,2)$ \\
\hline Mayor de 5 años & $74(60,1)$ \\
Índice de masa corporal & $22(18)$ \\
Normal & $55(46)$ \\
Sobrepeso & $43(36)$ \\
Obesidad & \\
Control glicémico & $36(30)$ \\
Buen control & $84(70)$ \\
\hline Mal control &
\end{tabular}

un tiempo de enfermedad mayor a 5 años, se observó IMC normal en $18 \%$ de participantes y solo $30 \%$ tuvo buen control glicémico. En la Tabla 2 se muestran los datos sociodemográficos y clínicos.

Con relación del nivel de actividad física, se halló que $20 \%$ de los pacientes calificaba como inactivo, $68 \%$ realizaban mínima actividad y $12 \%$ actividad física adecuada. La frecuencia de los niveles de actividad física se comparó con el tiempo de enfermedad $(p=0,68)$, control glicémico $(p=0,298)$ y el índice de masa corporal, categorizado $(p=0,325)$ sin encontrar diferencia significativa (Tabla 3 ).

El gasto energético expresado en MET-min/semana en pacientes con buen y mal control glicémico, arrojó medianas de 5046 MET-min/semana y 2936 MET-min/semana, sin que estas medianas fueran diferentes de manera significativa. Las medianas del gasto energético, en los grupos de buen o mal control glicémico, también fueron comparadas según cada dominio de la actividad física y tampoco se encontró diferencias, en los dominios de transporte $(p=0,407)$, trabajo $(p=0.94)$, hogar $(p=0,647)$ ni ocio $(p=0,862)$. En general, el gasto energético realizado fue mayor en las actividades de transporte y hogar, y menor en las actividades de ocio.

Tabla 3. Frecuencia de actividad física según control glicémico e IMC en pacientes con diabetes atendidos ambulatoriamente en el Hospital Nacional Cayetano Heredia $(n=120)$

\begin{tabular}{|c|c|c|c|c|c|c|}
\hline \multirow{2}{*}{$\begin{array}{l}\text { Nivel de actividad } \\
\text { física }\end{array}$} & \multicolumn{2}{|c|}{ Control glicémico } & \multicolumn{3}{|c|}{ Estado nutricional } & \multirow[b]{2}{*}{ Total (\%) } \\
\hline & Bueno (\%) & Malo (\%) & Normal (\%) & Sobrepeso (\%) & Obesidad (\%) & \\
\hline Inactivo & $7(19)$ & $17(20)$ & $4(18)$ & $12(22)$ & $818)$ & $24(20)$ \\
\hline Mínimamente activo & $27(75)$ & $54(65)$ & $15(68)$ & $36(66)$ & $30(70)$ & $81(68)$ \\
\hline Activo & $2(6)$ & $13(15)$ & $3(14)$ & $7(12)$ & $5(12)$ & $15(12)$ \\
\hline Total & $36(30)$ & $84(70)$ & $22(18)$ & $55(46)$ & $43(36)$ & $120(100)$ \\
\hline
\end{tabular}

Se realizó regresión lineal múltiple y se eliminó dos valores atípicos, se encontró que la edad fue el único factor que se asoció, con la actividad física, MET$\min /$ semana $(p=0,006)$.

\section{DISCUSIÓN}

Se encontró una elevada frecuencia de inactividad y de actividad mínima en pacientes diabéticos (88\%), porcentaje mayor al descrito en la población general a nivel mundial $(70 \%)^{(7,8)}$ y al encontrado por el INS y el CENAN entre el 2009 y el 2010, usando también la versión larga del IPAQ $(70,9 \% \text { de inactividad })^{(15)}$. Si bien estos estudios no estuvieron enfocados en pacientes con DM 2, la herramienta de medición fue la misma, lo que sugiere que los pacientes con DM 2 tendrían niveles mayores de inactividad y actividad física mínima que la población general.

Sí comparamos la frecuencia de actividad, 20\% inactivos, $68 \%$ mínimamente activos y $12 \%$ de activos, con la descrita por la serie de casos peruana publicada el 2011, por Zhang-Xu ${ }^{(16)}$, donde reportaron un $30 \%$ de inactividad, $31,2 \%$ de actividad mínima y $35,71 \%$ de actividad física adecuada, vemos que nuestros pacientes realizan menor actividad física, esta diferencia podría corresponder al tipo de muestreo. Mientras en el presente estudio utilizamos un modelo aleatorio, en el estudio referido, la muestra fue no probabilística. Sin embargo, en ambos estudios se nota una baja frecuencia de actividad física, lo cual resulta preocupante, pues todas las recomendaciones relacionadas con el cuidado de diabéticos, y en general para la preservación de la salud de la población, se enfocan en promover la realización de actividad física ${ }^{(7,8)}$.

No se encontró relación entre el control metabólico de los pacientes con DM 2 y la actividad física, lo que podría deberse a la combinación de bajo nivel de "control glicémico adecuado" en nuestra muestra y a la baja frecuencia de actividad física, en particular la asociada a las actividades de ocio, que son las que han demostrado tener un efecto protector en la salud (17). En los pacientes con DM 2 este efecto protector se ha demostrado con 
la disminución de la resistencia a la insulina, mejor control metabólico, disminución de la presión arterial, preservación de la masa muscular, reducción de la grasa corporal, promoviendo la pérdida del exceso de peso $\mathrm{y}$, consecuentemente, disminuyendo el riesgo cardiovascular ${ }^{(3,4,17)}$.

Se encontró una alta frecuencia de sobrepeso y obesidad $(82 \%)$, situación alarmante y lamentablemente compartida en Brasil $(87 \%)$ y Colombia $(71 \%)^{(18,19)}$. Cerca al $70 \%$ de los pacientes con sobrepeso y obesidad son activos o mínimamente activos, pero en dominios que no reditúan beneficiosos para la salud. Este menor nivel de actividad protectora de la salud ha sido explicada en países de medianos y bajos ingresos como el nuestro, por la poca realización de actividad física en el tiempo libre, el uso de vehículos motorizados como principal medio de transporte, aumento de la mecanización en el ámbito laboral y doméstico y el escaso tiempo de ocio ${ }^{(6-8)}$. Varios estudios han tratado de asociar obesidad y baja actividad física, sin embargo, no han logrado establecer ninguna relación ${ }^{(10)}$, en este estudio tampoco se encontró una asociación significativa entre el IMC y la actividad física.

Concordante con la literatura, se halló que existe una relación negativa entre la actividad física y la edad ${ }^{(8)}$, esta relación se comprueba también en los pacientes con DM 2, pero es independiente de la existencia o no de la enfermedad. No se halló correlación entre tiempo de enfermedad y la actividad física. Como se aprecia en la Tabla 2, más del $60 \%$ de pacientes tenían diagnostico mayor a 5 años por lo que no se pueden atribuir la inactividad física a escaso contacto con educación en salud o con personal de salud, pues la recomendación de realización obligatoria de actividad física forma parte de las indicaciones y recomendaciones que se dan a todo paciente con DM 2

Entre las limitaciones que se puede mencionar está el tamaño de la muestra que podría estar condicionando la no asociación de algunas variables, el haber tomado la muestra de un solo hospital, que limita la generalización de los resultados, además las características clínicas de nuestra muestra, es decir, la baja frecuencia de buen control glicémico en los pacientes con DM 2, podría haber interferido en la no asociación encontrada entre el control glicémico y la actividad física.

Existen niveles elevados de inactividad y de mínima actividad en pacientes con DM 2, siendo menor el gasto energético, precisamente en las actividades relacionadas al ocio que son las actividades beneficiosas para la salud. Igualmente, se constata que conforme aumenta la edad disminuye la actividad física realizada. Consideramos que se deberían investigar los motivos por los cuales los pacientes con DM 2 no realizan actividad física para implementar intervenciones que fomenten la actividad física en personas con DM2 y en la población general.

Conflictos de interés: los autores declaran no tener conflictos de interés.

Fuentes de financiamiento: autofinanciada.

\section{REFERENCIAS BIBLIOGRÁFICAS}

1. Wild S, Roglic G, Green A, Sicree R, King H. Global prevalence of diabetes: estimates for the year 2000 and projections for 2030 . Diabetes Care. 2004 May;27(5):104753.

2. Revilla L. Situación de la vigilancia de diabetes en el Perú, al I semestre de 2013. 2013;22(39):825-8.

3. Fauci A, Braunwald E, Kasper D, Hauser S, Longo D, Jameson JL. Harrison: Principles of Internal Medicine. 17 ed. McGraw-Hill. 2009.

4. Gaude P, Vedel P, Larsen N, Jensen GV, Parving HH, Pedersen O. Multifactorial interventions and cardiovascular disease in patients with type 2 diabetes. N Engl J Med. 2003 Jan 30;348(5):383-93.
5. Worl Health Organization. World Health Statistics 2012. Geneva: WHO; 2012

6. Serón P, Muñoz S, Lanas F. Nivel de actividad física medida a través del cuestionario internacional de actividad física en población chilena. Rev Med Chil. 2010 Oct;138(10):1232-9. doi: /S003498872010001100004 .

7. Worl Health Organization. World Health Report: reducing risk, promoting healthy life. Geneva: WHO; 2002.

8. Jacoby E, Bull F, Neiman A. Cambios acelerados del estilo de vida obligan a fometar la actividad física como prioridad en la región de las Américas. Rev Panam Salud Pública. 2003;14:223-5.
9. Lanas F, Avezum A, Bautista LE, Díaz $\mathrm{R}$, Luna M, Islam S, et al. Risk factors for acute myocardial infarction in Latin America: the INTERHEART Latin American study. Circulation. $2007 \mathrm{Mar}$ 6;115(9):1067-74.

10. As-da-Costa JS, Hallal PC, Wells JC, Daltoe T, Fuchs SC, Menezes AM, et al. Epidemiology of leisure-time physical activity: a population-based study in southern Brazil. Cad Saude Publica. 2005 Jan-Feb;21(1):275-82.

11. Brown WJ, Trost SG, Bauman A, Mummery K, Owen N. Test-retest reliability of four physical activity measures used in population surveys. J Sci Med Sport. 2004 Jun; 7 (2):205-15.

12. Lopez Stewart G, Tambascia M, Rosas Guzmán J, Etchegoyen F, Ortega Carrión J, Artemenko S. Control of 
type 2 diabetes mellitus among general practitioners in private practice in nine countries of Latin America. Rev Panam Salud Publica. 2007 Jul;22(1):12-20.

13. International Physical Activity Questionnaire [Internet]. IPAQ Group [citado el 07 de enero del 2014]. Disponible en: http://www.ipaq.ki.se/

14. World Health Organization. Managing the global epidemic of obesity. Geneva: WHO, 1997.

15. Instituto Nacional de Salud, Centro Nacional de Alimentación y Nutrición. Estado Nutricional en el Perú [Informe]. Componente nutricional ENAHO - CENAN julio 2009 - junio 2010. Lima: INS; 2011.
16. Zhang-Xu A, Vivanco M, Zapata F, Málaga G, Loza C. Actividad física global de pacientes con factores de riesgo cardiovascular aplicando el "International Physical Activity Questionaire (IPAQ). Rev Med Hered. 2011;22(3):115-20.

17. Popkin B. Urbanization, Lifestyle Changes and the Nutrition Transition. World Development. 1999;27(11):1905-16.

18. Carolino ID, Molena-Fernandes CA, Tasca RS, Macon SS, Cuman RK. Risk factors in patients with type 2 diabetes mellitus. Rev Lat Am Enfermagem. 2008 MarApr;16(2):238-44.
19. Alayón AN, Altamar-López D, Banquez-Buelvas C, Barrios-López K. Complicaciones crónicas, hipertensión y obesidad en pacientes diabéticos en Cartagena, Colombia. Rev Salud Publica (Bogota). 2009 Dec;11(6):857-64.

Correspondencia: Germán Málaga

Dirección: Calle las Cantutas 479, Casuarinas Sur, Santiago de Surco, Lima 33 Teléfono: +51992768300

Correo electrónico: germanmalaga@upch.pe

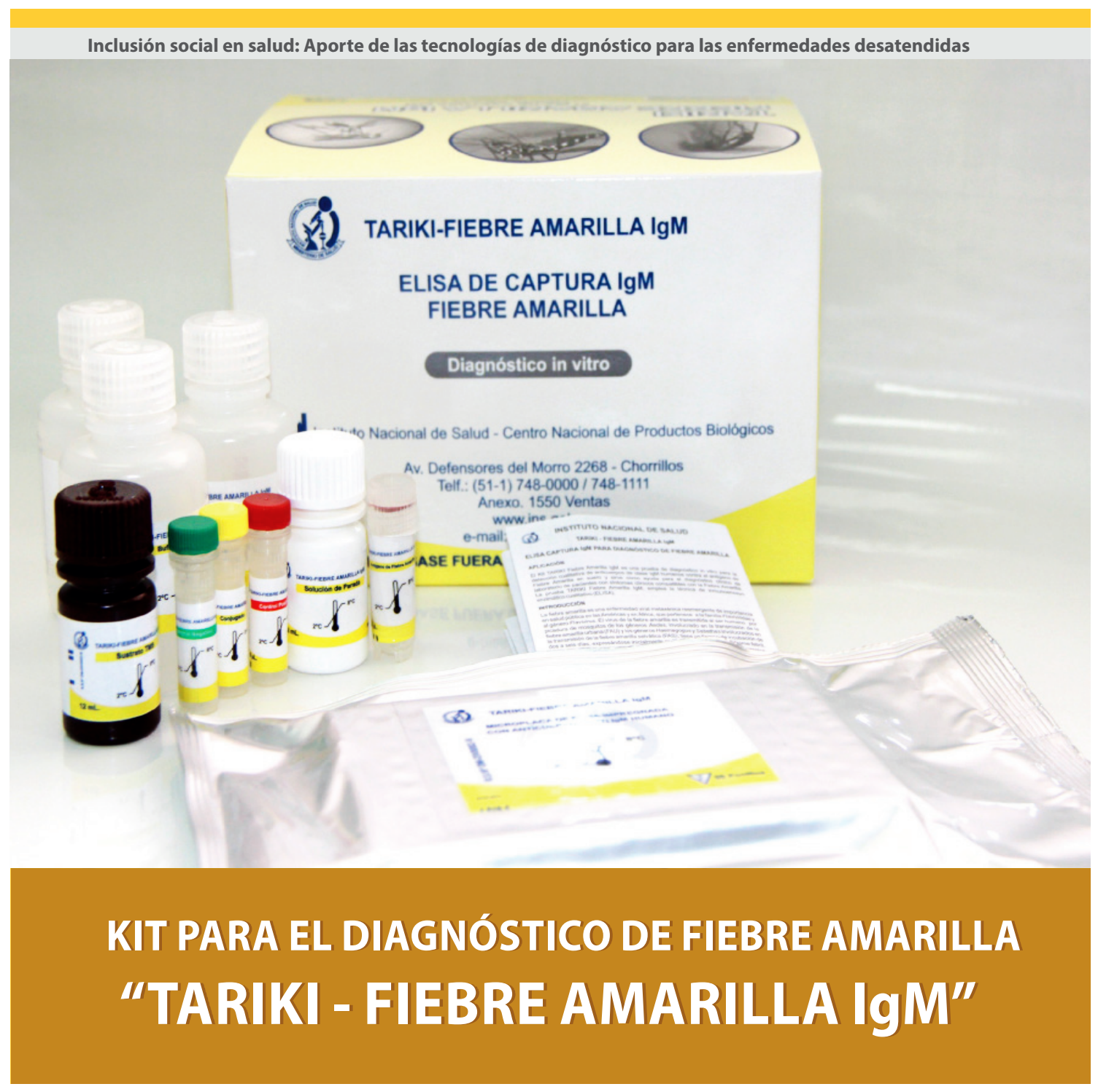

